\title{
Anterior uveitis and its relation to stress
}

\author{
B Mulholland, M Marks, S L Lightman
}

\begin{abstract}
Backgroundlaims-Idiopathic recurrent acute anterior uveitis (RAAU) is a common reason for attendance at ophthalmic casualty departments. Patients who suffer with this condition may have multiple recurrent episodes which are often debilitating and necessitate time off work. If recurrences are identified early and treatment initiated rapidly, the inflammation can be minimised and settles quickly on topical treatment with a speedy functional recovery by the patient. It has been our clinical impression that some patients with RAAU relate recurrences of inflammation to psychological factors. Therefore stress could be a trigger factor for recurrence, and at such times, patients should be alert to the early signs of uveitis and seek prompt examination by an ophthalmologist. This study tests the hypothesis that stressful life events are associated with the onset of RAAU.
\end{abstract}

Methods-The number of stressful life events in patients presenting to the casualty department with RAAU in the month immediately preceding symptom onset was compared with a control group of patients with other ocular problems taken from the casualty department using the Holmes and Rahe scale and the Spielberger state-trait anxiety inventory. Results-35 patients with RAAU did not report a higher number of stressful life events or higher levels of anxiety compared with 29 controls in this study on both Holmes and Rahe scale and Spielberger state-trait anxiety inventory. Scores for both groups were comparable with those published for normal adults of the same age and sex.

Conclusion-Stress was not shown in this study to be involved in triggering the recurrence of idiopathic acute anterior uveitis.

(Br f Ophthalmol 2000;84:1121-1124)

The involvement of stress in the incidence of disorders such as peptic ulcer, hypertension, and heart disease has been demonstrated. ${ }^{12}$ It is estimated that emotional stress plays an important part in more than $50 \%$ of diseases ${ }^{3}$ and many patients associate recurrences of uveitis with stressful life events. Duke-Elder recognised a relation between uveitis and psychological factors, ${ }^{4}$ and a connection with menstruation has also been described.

Some physical and emotional disorders tend to cluster around periods of major change in individuals' lives (such as divorce or moving house), and an attempt has been made to evaluate the degree of stress associated with various life events, with the Holmes and Rahe social readjustment rating scale. ${ }^{36}$ This scale has been developed over many years and quantitates various life events and the amount of coping required to deal with them. It takes the form of a list of events (see Table 1) which the patient is asked to look at and indicate if any have happened to them in the time before this presentation. ${ }^{7}$ For example, in a study where the role of life events in suicide attempts was examined using this rating scale, the mean social readjustment index was 255.2 (SD 137.01), which is a much higher score than that observed in other medical inpatients (180.69 (72.03)). ${ }^{8}$ The Spielberger state-trait anxiety inventory has been used extensively in research and clinical practice and has specifically been designed to measure the anxiety component of stress. ${ }^{9}$ It comprises separate self report scales for measuring state (S-anxiety) and trait (T-anxiety) anxiety. The S-anxiety scale consists of 20 statements that evaluate how respondents feel "right now at this moment" and evaluates feelings of apprehension, tension, nervousness, and worry. It has been used extensively to assess the levels of anxiety induced by unavoidable real life stressors such as interviews, exams, hospital visits, etc. ${ }^{10}$

The T-anxiety scale consists of 20 statements that assess how people generally feel before this time and has been widely used in assessing clinical anxiety in medical, surgical, psychosomatic, and psychiatric patients. ${ }^{10} 11$ The two scales are printed on the opposite sides of a single page test form.

The purpose of this study was to assess whether recurrent episodes of inflammation in patients with idiopathic acute anterior uveitis are related to life events and anxiety occurring in the 1 month before symptom onset.

Table 1 Examples of events and scores on social readjustment scale

\begin{tabular}{llc}
\hline Rank & Life event & Mean value \\
\hline 1 & Death of spouse & 100 \\
2 & Divorce & 73 \\
4 & Jail term & 63 \\
5 & Death of close family member & 63 \\
7 & Marriage & 50 \\
8 & Fired at work & 47 \\
10 & Retirement & 45 \\
12 & Pregnancy & 40 \\
17 & Death of a close friend & 37 \\
23 & Son or daughter leaving home & 29 \\
30 & Trouble with boss & 23 \\
31 & Change in work hours or & 20 \\
& conditions & \\
32 & Change in residence & 20 \\
41 & Vacation & 15 \\
42 & Christmas & 12 \\
\hline
\end{tabular}


Table 2 Age, sex distribution, and questionnaire scores of casualty controls compared with uveitis cases

\begin{tabular}{lll}
\hline & Casualty controls & Uveitis cases \\
\hline Number & 29 & 35 \\
Average age (years) & 37.3 & 44.36 \\
Male:female & $1.4: 1$ & $1.33: 1$ \\
Average number of life events & 2.76 & 2.02 \\
Average life event score & 80.1 & 67.54 \\
Average STAI-S score & 36.31 & 36.86 \\
Average STAI-T score & 38.1 & 36.77 \\
\% patients who felt stressed & $48 \%$ & $54 \%$ \\
\% female patients who felt recurrence was & $0 \%$ & $6.7 \%$ \\
$\quad$ related to menstrual cycle & & \\
\hline
\end{tabular}

Table 3 STAI anxiety level scores for published normal controls compared with uveitis cases aged 40-49 years (mean scores: males and females (SD))

\begin{tabular}{lllll}
\hline & Control S & Uveitis $S$ & Control T & Uveitis T \\
\hline Male & $35.88(10.52)$ & $27.5(6)$ & $35.06(8.88)$ & $34.5(8)$ \\
Female & $36.03(11.07)$ & $37.5(7.5)$ & $35.03(9.31)$ & $36.5(9)$ \\
\hline
\end{tabular}

Table 4 STAI anxiety level scores for published normal controls compared with casualty cases aged 50-69 years (mean scores: males and females (SD))

\begin{tabular}{lllll}
\hline & Control $S$ & Uveitis $S$ & Control $T$ & Uveitis $T$ \\
\hline Male & $34.51(10.34)$ & $35.5(6)$ & $33.86(8.86)$ & $32.5(4)$ \\
Female & $32.20(8.67)$ & $38(6)$ & $31.79(7.78)$ & $41(11)$ \\
\hline
\end{tabular}

\section{Subjects and methods}

Patients presenting to the casualty department at Moorfields Eye Hospital were recruited prospectively. Patients with recurrent episodes of acute anterior uveitis (RAAU) and controls (with corneal foreign bodies, conjunctivitis, and contact lens related disease) were invited to complete a questionnaire at the time of presentation to casualty. All patients were told on presentation to the casualty desk that a voluntary questionnaire based study was being conducted to assess whether stress and anxiety played a part in the onset of their eye condition and of any recurrences they had

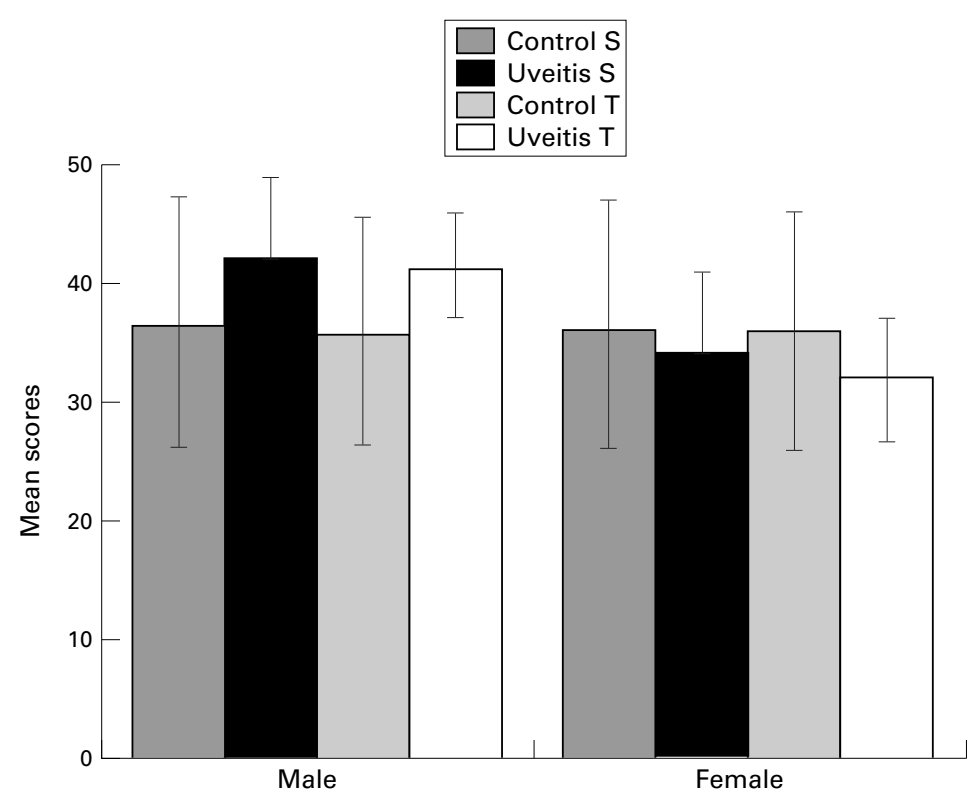

Figure 1 STAI anxiety level scores for published normal controls compared with uveitis cases aged 19-39 years. (Error bars = standard deviations.) experienced. They were invited to take part and were specifically asked to report any stressful life events (as listed on the Holmes and Rahe scale), and to complete the STAI questionnaire. Patients were then asked to report on the form if they felt they had been under increased stress in the month preceding symptom onset.

The Holmes and Rahe social readjustment rating scale was used to measure stress in terms of life events occurring in the month before symptom onset. ${ }^{6}$ The STAI was used to assess the T-anxiety and the S-anxiety level and is being used in this study to assess whether individuals are under increased stress now (state) relative to their normal "basal level" or trait. ${ }^{9}$

Patients were specifically asked whether they had been under increased stress in the month before symptom onset and females with RAAU were asked whether any recurrences were related to the menstrual cycle.

\section{Results}

Thirty five patients with RAAU (average age 44.36 years, male:female $1.33: 1$ ), and 29 controls, with corneal foreign bodies, conjunctivitis, and contact lens related disease (average age 37.3 years male:female 1.4:1) volunteered to take part in the study. Only two patients declined to participate.

Sufficient patients were recruited for the study to detect a clinically significant difference between the control and RAAU patients groups if it occurred. However, patients with RAAU were not found to have a higher number of stressful life events on the Holmes and Rahe scale or a higher anxiety level (on Spielberger analysis) at the time of symptom onset, when compared as a whole with the control group of casualty patients (Table 2).

Results for the STAI are presented separately (Tables 3 and 4 and Figures 1 and 2), compared with the published normals for each age group. ${ }^{9}$ Thirty five uveitis patients and 29 controls showed no significant difference, when analysed by age group, with $T$ and $S$ scores comparable with those published for normal adults of the same age and sex (unpaired $t$ test, $\mathrm{p}>0.1)^{7}$ (Tables 3 and 4). However, there was a trend towards increased stress in measurement with the Spielberger STAI in males aged 19-39 years (Fig 1). The STAI scores of the casualty control group aged 19-39 years were very similar to those published for normal subjects of the same age (Fig 2). ${ }^{9}$

Forty eight per cent of controls and $54 \%$ of uveitis patients reported subjectively increased stress during the month before presentation, as measured by subjective report on questioning, which was not significantly different but this is not a validated measure. An association between the menstrual cycle and RAAU was reported in one individual (see Table 2). Thirty three per cent of women with uveitis in this study were postmenopausal. 


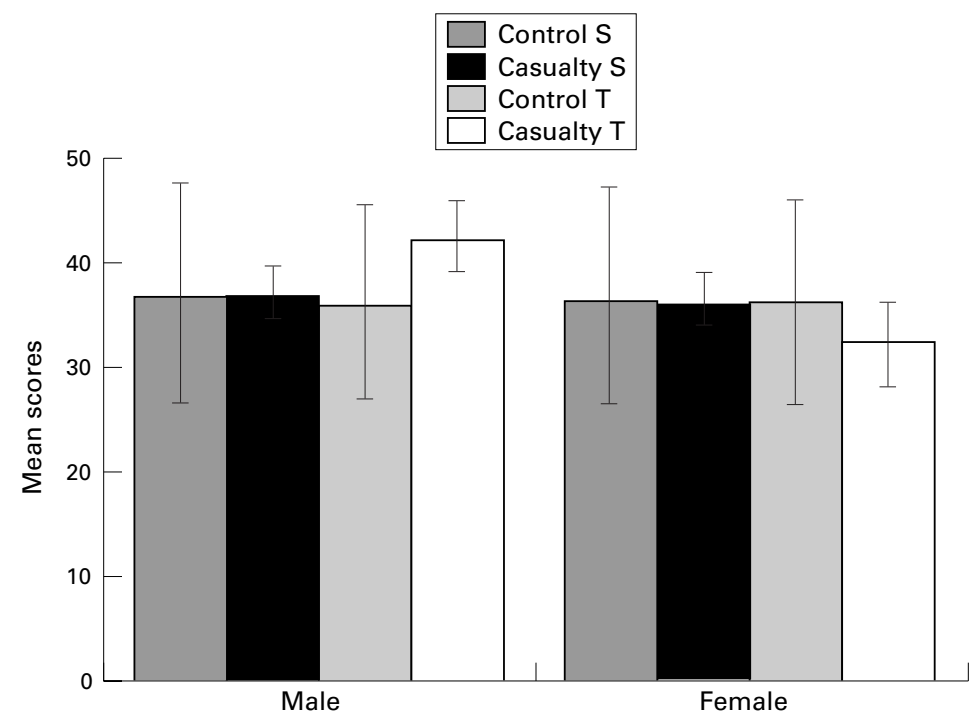

Figure 2 STAI anxiety level scores for published normal controls compared with casualty controls aged 19-39 years. (Error bars = standard deviations.)

\section{Discussion}

Stressful life events and psychological distress were not related to relapses of idiopathic RAAU in the present study and this was consistent with previous studies in the literature. ${ }^{12}{ }^{13}$ Secchi et $a l^{12}$ looked at 60 patients with idiopathic anterior uveitis, 15 with a recurrence (mean age 43.3, M:F 1.1:1), and 45 with quiet eyes (mean age $40.9 \mathrm{M}: \mathrm{F}$, $1.1: 1$ ) and 60 controls with medical and not ocular disease. Using the Paykel scale to evaluate life events in a quantitative and qualitative fashion in the preceding 12 months, there was no statistical difference between the two groups of patients with uveitis or between those with uveitis and the controls. There was also no statistically significant difference using a symptom distress check list despite a higher score in the group of 15 with recurrent uveitis.

Another study looked at 48 cases $(\mathrm{M}: \mathrm{F}$, 1.52), of recurrent uveitis (anterior and posterior). ${ }^{13}$ No recognised stress or life event questionnaire was used, although subjects were interviewed by the same interviewer using specific questions. In $37.5 \%$ of cases, they found that stress had a direct bearing on development or progression of uveitis-in $16.6 \%$ there was judged to be an acute strain in interpersonal relationships-and concluded that stress was found to precipitate recurrence in some cases. Acute stressful episodes were implicated rather than chronic continued stress. Future research may be important to distinguish between acute and chronic stressful life events as subjects may be more prone to illness after chronic stress than acute.

Kumar et al performed biochemical evaluation in patients with uveitis and reported a definite relation between stress index and bioamines (catecholamines, adrenaline, and noradrenaline) and whole blood histamine levels. A modified life inventory scale was used to assess the 6 months preceding the onset on symptoms and a definite correlation was reported between stress scores and uveitis. ${ }^{14}$
In view of the varied reports in the literature and the clinical impression that there is a correlation, we felt the relation of stress to RAAU deserved further study. The aim of this study was to formally test this association using well established methods. The results of this study, however, have not shown that stress is involved in triggering the recurrence of idiopathic acute anterior uveitis, using the life event table of Holmes and Rahe $^{6}$ and a recognised stress questionnaire, the Spielberger state-trait anxiety inventory. ${ }^{9}$ However, there does appear to be a trend towards increased stress measurement with the Spielberger STAI in males aged 19-39 years $(p<0.1)$. Although the study numbers are small, enough patients were recruited to be able to detect clinically significant differences (defined as more than $50 \%$ of patients having a medium anxiety score of $40-59$ with this occurring in less than $10 \%$ of the control group) should they have occurred. The same number of life events occurred in the month before casualty department attendance in the control and uveitis groups and the life event score was similar. This suggests that they were the same type of events which had occurred as the scoring is weighted so that more major events such a bereavement have much higher scores than less significant events (see Table 1).

It is interesting to speculate why our results are different from those previously reported in the literature. It may be that there is a significant difference in the cultural mix of the subjects taking part in this study as previous studies were performed in the USA rather than in the UK, although the STAI scores of the casualty control group was very similar to the published scores in the literature for the age range 19-39 years. ${ }^{9}$ Also the use of a different validated stress questionnaire to measure anxiety levels may have influenced the findings.

The trend towards increased anxiety in male patients with RAAU aged 19-30 years is interesting. However, the STAI scale only measures anxiety as one component of stress and broader range of symptoms may be analysed using, for example, the general health questionnaire. ${ }^{15}$ This might highlight a larger difference between RAAU sufferers and controls. Many of the patients in the control group had ocular problems of a recurrent nature, such as recurrent erosions, recurrent conjunctivitis, blepharitis, or corneal foreign bodies. The number of recurrences was not matched, which may be a confounding factor as psychological morbidity may be due to recurrent disease. Chronic minor stressful events could also play a part as could depression. ${ }^{11}$

Presented in part at the annual meeting of the Association of Research into Vision and Ophthalmology, Fort Lauderdale, Florida, USA.

1 Rahe RH, Benett C. Recent life changes and coronary heart disease in Finland. Am f Psychiatry 1973;110:1212.

2 Gray SJ, Benson JN, John A, et al. Chronic stress and peptic ulcer. $\mathscr{f} A M A$ 1957;147:1529-37.

3 Atkinson RL. Conflict and stress. In: Introduction to psycholAtkinson RL. Conflict and stress. In: Introduction to psychol-
ogy. Harcourt Brace Jovanovich International Edition. (eighth edition):438-49.

4 Duke-Elder S. Diseases of the uveal tract. In: System of ophthalmology. London: Henry Kimpton, 1966;Vol IX:85-8. 
5 Heaton JM. An ecologic approach to uveitis. Am $\mathcal{f}$ Ophthalmol 1964;17:122-8.

6 Holmes TH, Rahe RH. The social readjustment rating scale. F Psychosom Res 1967;11:213-18.

Smith CK, Cullison SW, Polis E, et al. Life change and illness onset: importance of concepts for family physicians. f Fam Pract 1978;7:975-81

8 Vizan Ferrero R, Benitez MH, Gracia Marco R, et al. Life events and self-destructive behavior. Actas Luso Esp Neurol Psiquiatr Cienc Afines 1993;21:205-10.

9 Spielberger CD. State-trait anxiety inventory for adults. Palo Alto, CA: Consulting Psychologists Press Inc, 1983.

10 Speilberger CD. State-trait anxiety inventory: a comprehensive bibliography. 2nd ed. Palo Alto, CA: Consulting Psychologists Press, 1989
11 Addolorato G, Capristo E, Stefanini GF, et al. Inflammatory bowel disease: a study of the association between anxiety and depression, physical morbidity, and nutritional status. Scand $\mathcal{F}$ Gastroenterol 1997;32:1013-21.

12 Secchi AG, Magni G. Tognon MS, et al. A psychosomatic approach to idiopathic recurrences of anterior uveitis. $A m \mathcal{F}$ Ophthalmol 1987;104:174-8.

13 Sen DK. Endogenous uveitis in relation to stress-a clinical study. Eye Ear Nose and Throat Monthly 1971; 50:108-10.

14 Kumar A, Nema HV, Thakur V. Stress and uveitis. Ann Ophthalmol 1981;13:1077-80.

15 Goldberg DP, Hillier VF. A scaled version of the general health questionnaire. Psychol Med 1979;9:139-45. 